\title{
含噁二唑结构的甲氧基丙烯酸酯类杀菌剂的合成及生物活性研究
}

刘阳刘明陈明桂
王宝雷 吴正名*

(南开大学元素有机化学研究所 元素有机化学国家重点实验室 天津化学化工协同创新中心 天津 300071)

\begin{abstract}
摘要 基于生物电子等排和活性亚结构拼接原理, 设计合成了 16 个结构新颖, 含噁二唑结构的甲氧基丙烯酸酯类杀菌 剂, 化合物结构均已通过核磁共振氢谱、碳谱和高分辨质谱确证, 并对其进行了离体杀菌活性测试. 结果表明: 部分化 合物对于油菜菌核病菌的活性与对照药嘧菌酯相当, 部分化合物对于小麦纹枯病菌活性超过对照药嘧菌酯. 进行了初 步的构效关系研究. (E)-2-\{2-[4-(噁二唑-2-基)苯基硫甲基]苯基 $\}$-3-甲氧基丙烯酸甲酯(IIa)和 $(E)$-2-\{2-[4-(5-乙基噁二唑2-基)苯基硫甲基]苯基\}-3-甲氧基丙烯酸甲酯(IIc)对于多种真菌表现很好的抑制活性，可作为先导化合物进一步研究. 关键词 1,3,4-慧二坐; 甲氧基丙烯酸酯类杀菌剂; 离体; 杀菌活性
\end{abstract}

\section{Design, Synthesis and Bioactivities of Novel Strobilurin Derivatives Containing 1,3,4-Oxadiazole Moity}

\author{
Liu, Yang Liu, Ming Chen, Minggui Wu, Changchun Hua, Xuewen Zhou, Sha \\ Wang, Baolei Li, Zhengming*
}

(Collaborative Innovation Center of Chemical Science and Engineering (Tianjin), State Key Laboratory of Elemento-Organic Chemistry, Nankai University, Tianjin 300071)

\begin{abstract}
A series of novel strobilurin derivatives containing 1,3,4-oxdiazole moity were designed and synthesized based on the bioisosterism and coordination of active substructure theories. The structures of new compounds were characterized by ${ }^{1} \mathrm{H}$ $\mathrm{NMR},{ }^{13} \mathrm{C}$ NMR and HRMS spectra. The bioassay indicated that the fungicidal activities of some compounds against Sclerotinia sclerotiorum reached the activity of azoxystrobin as the control and some compounds against Rhizotonia cerealis in vitro were more effective than the same control. Their structure-activity relationship was discussed. Methyl $(E)-2-(2-((4-(1,3,4-$ oxadiazol-2-yl)phenoxy)methyl)phenyl)-3-methoxyacrylate (IIa) and methyl (E)-2-(2-((4-(5-ethyl-1,3,4-oxadiazol-2-yl)phenoxy)methyl)phenyl)-3-methoxyacrylate (IIc) could be considered as leading compounds for further investigation.

Keywords 1,3,4-oxdiazole; strobilurin; in vitro; fungicidal activity
\end{abstract}

$\beta$-甲氧基丙烯酸酯类杀菌剂是从天然产物 Strobilurin A 以及 Oudenemansin A 经过 20 多年的结构 优化发展而来, 具有高效、广谱、对哺乳动物低毒和对 环境友好等特点 ${ }^{[1]}$. 从已仿生研发成功的甲氧基丙烯酸 酯类杀菌剂来看, 其结构可以分为三部分(图 1): 侧链、 芳香桥和药效团 ${ }^{[2]}$. 对于芳香桥与药效团的改动, 会影 响药效的发挥, 而对于侧链的改动却容易获得高活性化 合物 ${ }^{[3]}$. 杂环类化合物具有广泛的生物活性, 如抗细 菌 $^{[4 \sim 6]}$ 、抗炎症 ${ }^{[7]}$ 、抗病毒 ${ }^{[8]}$ 、抗真菌 ${ }^{[9-11]}$ 、抗肿瘤 ${ }^{[12,13]}$,

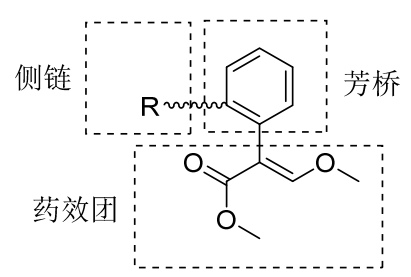

图 1 甲氧基丙烯酸酯类杀菌剂结构

Figure 1 The structure of fungicide $\beta$-methoxyacrylates

同时, 杂环结构可以优化化合物的动力学参数, 例如油

* Corresponding author. E-mail: nkzml@vip.163.com

Received July 19, 2016; revised September 12, 2016; published online October 18, 2016.

Project by the "111" Project of Ministry of Education of China (No. B06005), the State Key Laboratory of Elemento-organic Chemistry and Collaborative Innovation Center of Chemical Science and Engineering (Tianjin).

高等学校学科创新引智计划(No. B06005)、元素有机化学国家重点实验室和天津化学化工协同创新中心资助项目. 
水比、溶解性等 ${ }^{[14]}$. 也有报道噁二唑具有抗真菌活 性 ${ }^{[4,15]}$. 在许多具有杀菌活性的化合物中含有噁二唑结 构(图 2). 另外, 化合物中引入硫醚键可以提高化合物的 杀菌活性 ${ }^{[16,17]}$, 芳环之间通过供电子元素硫或氧相连, 利于供受体之间的结合, 从而提高药效 ${ }^{[18]}$. 硫元素具有 良好的杀菌活性，例如代森锰锌、福美双、福美锌、叶 枯灵中均含有硫元素. 亚砜基团具有广泛生物活性, 亚 砜类杀菌剂广泛存在于农药领域 ${ }^{[19]}$. 将硫原子和亚砜 结构分别与 1,3,4-嘒二唑连接可以产生具有良好杀菌活 性的化合物(图 2) ${ }^{[20 ~ 23]}$. 基于以上结论, 利用生物电子 等排和活性片段拼接的方法, 将噁二唑片段与芳香环连 接, 形成芳联杂环侧链; 通过硫原子或氧原子将芳联杂 环侧链与甲氧基丙烯酸酯药效团相连, 设计合成了一系 列未见文献报道的含噁二唑结构的甲氧基丙烯酸酯类 化合物, 以期获得高活性的杀菌剂先导化合物.

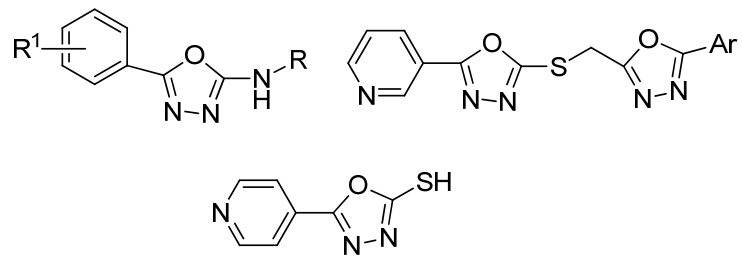

图 2 具有杀菌活性的 1,3,4-惡二唑类化合物

Figure 2 Fungicidal compounds containing 1,3,4-oxadia- zole moity

\section{1 结果与讨论}

\section{1 目标化合物的合成}

所设计化合物结构主要包含两部分, 左半部分为芳 环联噁二唑部分, 右半部分为甲氧基丙烯酸酯部分.

中间体 1 是由对应羧酸与甲醇发生酯化反应制备的 甲酯与水合肼在乙醇中发生肼解反应制得. 1 与二硫化 碳在氢氧化钾的乙醇溶液中回流合成 2,2 与 4 在 $\mathrm{K}_{2} \mathrm{CO}_{3} / \mathrm{DMF}$ 体系中发生亲核取代反应制备终产物 $\mathbf{I}$.

化合物 3 的合成参照文献[4]制备. 3 与溴代丁二酰 亚胺(NBS)发生苄位的溴代反应得到中间体 4. 需要注 意: 中间体 3 存在 $Z / E$ 异构体，在合成中间体 $\mathbf{4}$ 的过程 中, 由于条件比较剧烈, 发生自由基取代得到的产物构 型大部分为热力学稳定结构 $E$ 式.

化合物 II 是由 5 与原甲酸酯关环合成 6,6 与 4 在 $\mathrm{K}_{2} \mathrm{CO}_{3} / \mathrm{DMF}$ 体系中发生亲和取代反应制备.

化合物 III 是由系列 I氧化而来. 常用的氧化剂有间 氯过氧苯甲酸(MCPBA)、双氧水/二水合铇酸钠、次氯 酸钠、醋酸碘苯、酸性高锰酸钾. 酸性高锰酸钾的氧化 性过强, 反应不易控制, 容易过氧化生成砜类化合物. 经过探索之后, 发现 MCPBA 为最佳氧化剂.

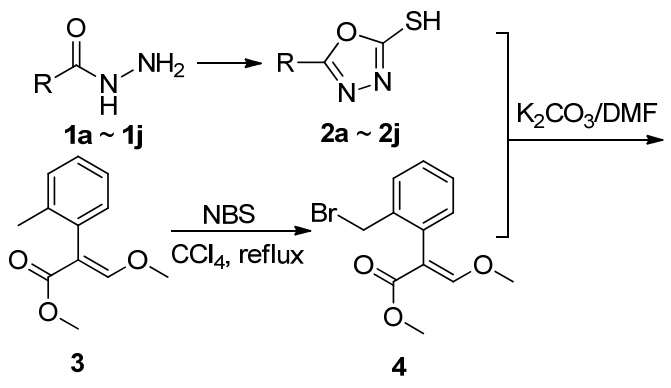<smiles>[R]c1nnc(SCc2ccccc2C(COC)C(=O)OC)o1</smiles>

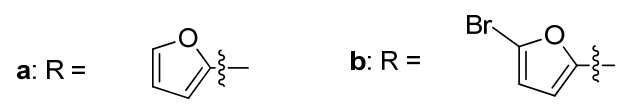

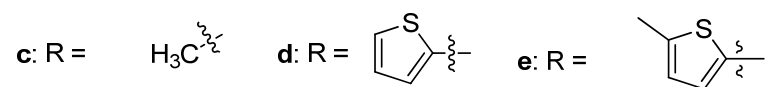<smiles>Cc1cccc(C)n1</smiles>

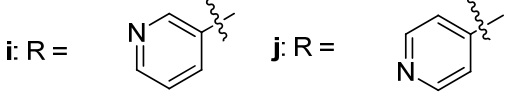

图式 1 化合物 I 和 III 的合成路线

Scheme 1 Synthetic route of compounds I and III

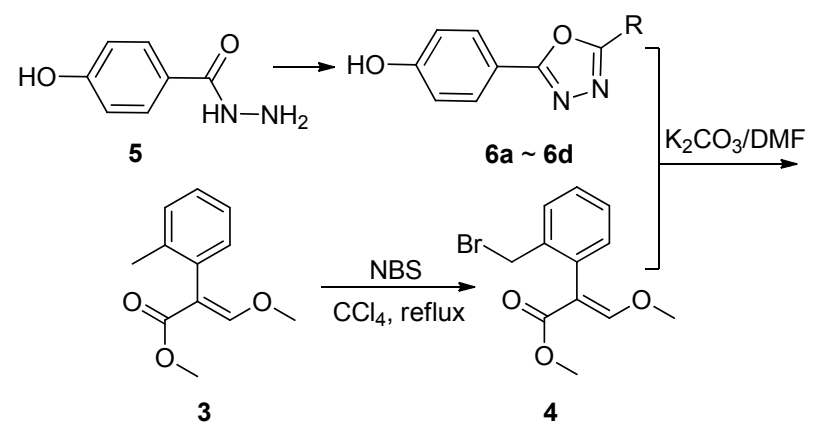<smiles>[R]c1nnc(-c2ccc(OCc3ccccc3/C(=C\OC)C(=O)OC)cc2)o1</smiles>

a: $\mathrm{R}=\mathrm{H} ; \mathbf{b}: \mathrm{R}=\mathrm{CH}_{3} ; \mathbf{c}: \mathrm{R}=\mathrm{CH}_{2} \mathrm{CH}_{3} ; \mathbf{d}: \mathrm{R}=\mathrm{Ph}$

图式2 化合物II的合成路线

Scheme 2 Synthetic route of compounds II

\section{2 生物活性测试结果}

目标化合物的离体抑真菌活性如表 1 所示. 结果表 明, 该系列化合物在 $50 \mathrm{mg} / \mathrm{L}$ 的测试浓度下, 对于苹果 轮纹病菌 (Physalospora piricola)、花生褐斑病菌 (Cercospora arachidicola Hori)、番茄早疫病菌 
(Alternaria solani)和黄瓜褐斑病菌(Corynespora cassiicola) 的活性一般.

表 1 目标化合物在 $50 \mathrm{mg} / \mathrm{L}$ 剂量下的离体杀菌活性(\%) ${ }^{a}$

Table 1 Fungicidal activities (\%) in vitro of target compounds at a concentration of $50 \mathrm{mg} / \mathrm{L}$

\begin{tabular}{ccccccc}
\hline Compd. & SS & PP & CH & RC & AS & CC \\
\hline Ia & 86.8 & 63.3 & 60.9 & 80.3 & 71.4 & 42.9 \\
Ib & 93.4 & 63.3 & 69.6 & 91.5 & 67.9 & 42.9 \\
Ic & 50.0 & 51.5 & 42.0 & 68.8 & 64.3 & 42.9 \\
Id & 81.5 & 50.6 & 51.2 & 94.2 & 60.7 & 57.1 \\
Ie & 82.7 & 60.2 & 46.5 & 95.3 & 64.3 & 42.9 \\
If & 86.4 & 55.4 & 46.5 & 93.0 & 64.3 & 50.0 \\
Ig & 83.8 & 56.6 & 53.5 & 78.4 & 60.7 & 35.7 \\
Ih & 90.2 & 41.0 & 41.9 & 60.5 & 64.3 & 50.0 \\
Ii & 80.0 & 51.8 & 48.8 & 80.2 & 64.3 & 50.0 \\
Ij & 80.2 & 44.6 & 41.9 & 73.3 & 71.4 & 50.0 \\
IIa & 100.0 & 59.0 & 46.5 & 98.8 & 67.9 & 50.0 \\
IIb & 98.8 & 43.4 & 39.5 & 97.7 & 71.4 & 42.9 \\
IIc & 100.0 & 60.2 & 48.8 & 87.2 & 67.9 & 57.1 \\
IId & 55.6 & 68.7 & 62.8 & 75.6 & 75.0 & 50.0 \\
IIIa & 51.5 & 49.1 & 42.0 & 64.7 & 64.3 & 50.0 \\
IIIb & 63.2 & 50.0 & 54.3 & 66.2 & 46.4 & 57.1 \\
嘧菌酯 & 96.3 & 62.7 & 51.2 & 70.9 & 64.3 & 57.1 \\
\hline
\end{tabular}

${ }^{a}$ SS: Sclerotinia sclerotiorum; PP: Physalospora piricola; $\mathrm{CH}$ : Cercospora arachidicola Hori; RC: Rhizotonia cerealis; AS: Alternaria solani; CC: Corynespora cassiicola.

目标化合物对于油菜菌核病菌(Sclerotinia sclerotiorum)和小麦纹枯病菌(Rhizotonia cerealis)表现出较高 的活性. Ib (91.5\%), Id (94.2\%), Ie (95.3\%), If (93.0\%), IIa(98.8\%), IIb (97.7\%), IIc (87.2\%)对于小麦纹枯病菌 的活性超过对照药嘧菌酯 (70.9\%). Ia (86.8\%), Ib (93.4\%), Ih (90.2\%), IIa (100\%), IIb (98.8\%), IIc (100\%) 对于油菜菌核病菌的活性与对照药嘧菌酯 $(96.3 \%)$ 相当. 呋喃、噻吩、吡啶等芳香基团有利于提升化合物对于油 菜菌核病和小麦纹枯病的活性, 用简单脂肪链取代芳香 基团与噁二唑相连会严重降低化合物整体药效的发挥. 而将苯环同时与噁二唑, 甲氧基丙烯酸酯芳桥相连更有 利于提升化合物的活性. 同时, 亚砜基团的引入降低了 整体的药效, 推测是由于亚砜基团属于吸电子基团, 降 低了相邻芳香环上的电子云密度, 导致活性大大下降. 作为侧链与芳香桥连接原子, 硫原子要优于氧原子. 推 测是由于体积更大的硫原子受两侧芳香环的影响较小, 同时硫原子可以提高芳香环的电子云密度.

进一步测试 IIa 和 IIc 对另外 8 种真菌黄瓜枯萎病 菌 (Fusarium oxysporum)、玉米小斑病菌 (Bipolaris maydis)、西瓜炭疽病菌(Colletotrichum orbiculare)、水 稻恶苗病菌 (Gibberella fujikuroi)、小麦赤霉病菌 (Fusarium graminearum)、马铃薯晚疫病菌(Phytophthora infestans)、番茄灰霉病菌(Botrytis cinerea)、水稻纹枯病
菌(Thanatephorus cucumers)的活性(表 2), 结果表明 IIa 和 IIc 对于黄瓜枯萎病菌、玉米小斑病菌、西瓜炭疽病 菌、水稻恶苗病菌、小麦赤霉病菌、马铃薯晚疫病菌、 番茄灰霉病菌、水稻纹枯病菌具有一定的活性. 综合针 对多种病菌的活性考虑, IIa 和 IIc 可以作为先导化合物 进行进一步研究.

表 2 目标化合物 IIa 和 IIc 在 $50 \mathrm{mg} / \mathrm{L}$ 剂量下的离体杀菌活 性 $(\%)^{a}$

Table 2 Fungicidal activities (\%) in vitro of compounds IIa and IIc at a concentration of $50 \mathrm{mg} / \mathrm{L}$

\begin{tabular}{ccccccccc}
\hline Compd. & FO & BM & CO & GF & FG & PI & BC & TC \\
\hline IIa & 37.0 & 31.1 & 33.3 & 53.3 & 53.6 & 30.0 & 41.7 & 30.9 \\
IIc & 40.7 & 49.2 & 54.2 & 56.7 & 44.6 & 50.0 & 33.3 & 32.1 \\
嘧菌酯 & 53.7 & 55.7 & 64.6 & 70.0 & 69.6 & 57.5 & 33.3 & 46.9 \\
\hline
\end{tabular}

${ }^{a}$ FO: Fusarium oxysporum; BM: Bipolaris maydis; CO: Colletotrichum orbiculare; GF: Gibberella fujikuroi; FG: Fusarium graminearum; PI: Phytophthora infestans; BC: Botrytis cinerea; TC: Thanatephorus cucumers.

分析 Ie $\left(\mathrm{EC}_{50}=9.196 \mathrm{mg} / \mathrm{L}\right)$ 和 If $\left(\mathrm{EC}_{50}=10.52\right.$ $\mathrm{mg} / \mathrm{L}$ )的活性与结构特征, 结果表明含给电子基团的噻 吩环有利于提高化合物对于小麦纹枯病菌的活性(表 3). Ia, Ib, Ih 与 IIa, IIb, IIc 相比表明, 硫原子作为连接基团 更有利于提高化合物对于油菜菌核病菌的活性(表 4). 分析 IIa, IIb, IIc 三个化合物的活性与结构特征表明, 含有简单烷基链的噁二唑环与苯环直接相连的结构更 有利于提高化合物对于油菜菌核病菌活性.

表 $3 \mathrm{Ib}, \mathbf{I d}, \mathbf{I e}, \mathbf{I f}, \mathbf{I I a}, \mathbf{I I b}, \mathbf{I I c}$ 对于 Rhizotonia cerealis 的 $\mathrm{EC}_{50}$ 值

Table 3 Further screen and $\mathrm{EC}_{50}$ values of Ib, Id, Ie, If, IIa, IIb, IIc against Rhizotonia cerealis

\begin{tabular}{cccc}
\hline Compd. & $R$ & $\mathrm{EC}_{50} /\left(\mathrm{mg} \cdot \mathrm{L}^{-1}\right)$ & 回归方程 \\
\hline Ib & 0.9958 & 10.05 & $y=1.6179 x+3.3786$ \\
Id & 0.9986 & 14.01 & $y=1.6897 x+3.0633$ \\
Ie & 0.9886 & 9.196 & $y=1.5704 x+3.4867$ \\
If & 0.9912 & 10.52 & $y=1.8185 x+3.1416$ \\
IIa & 0.9971 & 15.93 & $y=2.2559 x+2.2878$ \\
IIb & 0.9991 & 9.353 & $y=1.7414 x+3.3091$ \\
IIc & 0.9957 & 13.45 & $y=1.8883 x+2.8682$ \\
嘧菌酯 & 0.9987 & 22.86 & $y=1.6527 x+2.7539$ \\
\hline
\end{tabular}

\section{2 结论}

基于生物电子等排和活性亚结构拼接原理, 设计合 成了 16 个结构新颖、含惡二坐结构的甲氧基丙烯酸酯 类杀菌剂, 并对其进行了杀菌活性测试. 测试结果表明: 含有给电子基团的噻吩联噁二唑结构有利于提升化合 物对于小麦纹枯病菌的活性; 硫原子作为连接原子以及 含有简单烷基链的噁二唑与苯环直接相连的结构, 更有 利于提高化合物对于油菜菌核病菌的活性. IIa 和 IIc 对 
表 4 Ia, Ib, Ih, IIa, IIb, IIc 对于 Sclerotinia sclerotiorum 的 $\mathrm{EC}_{50}$ 值

Table 4 Further screen and $\mathrm{EC}_{50}$ values of Ia, Ib, Ih, IIa, IIb and IIc against Sclerotinia sclerotiorum

\begin{tabular}{cccc}
\hline Compd. & $R$ & $\mathrm{EC}_{50} /\left(\mathrm{mg} \cdot \mathrm{L}^{-1}\right)$ & 回归方程 \\
\hline Ia & 0.9969 & 5.4701 & $y=1.1096 x+4.1811$ \\
Ib & 0.9787 & 4.7308 & $y=1.3365 x+4.0979$ \\
Ih & 0.9967 & 5.2105 & $y=1.5381 x+3.8974$ \\
IIa & 0.9706 & 7.6694 & $y=2.4198 x+2.8590$ \\
IIb & 0.9714 & 7.3555 & $y=2.3097 x+2.9984$ \\
IIc & 0.9816 & 6.1591 & $y=2.2695 x+3.2082$ \\
嘧菌酯 & 0.9844 & 4.6775 & $y=1.5871 x+3.9366$ \\
\hline
\end{tabular}

于多种真菌均具有较好活性, 可作为先导化合物进行进 一步研究.

\section{3 实验部分}

\section{1 仪器与试剂}

X-4 数字显示显微熔点测定仪 (北京泰克仪器有限 公司), 温度未校正; Bruker Avance-400 MHz 核磁共振 仪; FTICR-MS (Varian 7.0T)型高分辨率质谱仪. 所用试 剂均为市售分析纯或化学纯. $\mathrm{CDCl}_{3}$ 或 DMSO- $d_{6}$ 购自百 灵威, TMS 为内标. 硅胶 $(200 \sim 300$ 目)为青岛海洋化工 厂产品. 对照药嘧菌酯购自上海将来试剂有限公司.

\section{2 实验方法}

\subsection{1中间体化合物 $\mathbf{2}$ 的合成}

以取代呋喃甲酸、取代噻吩甲酸、吡啶甲酸或乙酸 乙酯为原料, 按照文献[24]步骤合成化合物 $\mathbf{2}$ 的粗品.

5-(呋喃-2-基)-1,3,4-噁二唑硫醇(2a): 白色固体, 收 率 84\%. m.p. $170{ }^{\circ} \mathrm{C}$ (文献值 ${ }^{[25]} 166 \sim 167{ }^{\circ} \mathrm{C}$ ); ${ }^{1} \mathrm{H}$ NMR $\left(400 \mathrm{MHz}\right.$, DMSO- $\left.d_{6}\right) \delta: 14.81$ (bs, $\left.1 \mathrm{H}\right), 8.07(\mathrm{~s}, 1 \mathrm{H}), 7.36$ (d, $J=3.6 \mathrm{~Hz}, 1 \mathrm{H}), 6.92 \sim 6.67(\mathrm{~m}, 1 \mathrm{H})$. HRMS calcd for $\mathrm{C}_{6} \mathrm{H}_{5} \mathrm{~N}_{2} \mathrm{O}_{2} \mathrm{~S}\left([\mathrm{M}+\mathrm{H}]^{+}\right)$169.0066, found 169.0067.

5-(5-澳呋喃-2-基)-1,3,4-噁二唑硫醇 (2b): 白色固 体, 收率 86\%. m.p. 178 181 ${ }^{\circ} \mathrm{C} ;{ }^{1} \mathrm{H}$ NMR $(400 \mathrm{MHz}$, DMSO- $\left.d_{6}\right) \delta: 15.22(\mathrm{bs}, 1 \mathrm{H}), 7.55(\mathrm{~d}, J=3.6 \mathrm{~Hz}, 1 \mathrm{H}), 7.11$ (d, $J=3.6 \mathrm{~Hz}, 1 \mathrm{H})$. HRMS calcd for $\mathrm{C}_{6} \mathrm{H}_{4} \mathrm{BrN}_{2} \mathrm{O}_{2} \mathrm{~S}([\mathrm{M}+$ $\mathrm{H}]^{+}$) 246.9171, found 246.9172 .

5-甲基-1,3,4-噁二唑硫醇 (2c): 淡黄色固体, 收率 $82 \%$. m.p. $76{ }^{\circ} \mathrm{C}$ (文献值 ${ }^{[26]} 74 \sim 75{ }^{\circ} \mathrm{C}$ ); ${ }^{1} \mathrm{H}$ NMR $(400$ MHz, DMSO- $d_{6}$ ) $\delta: 14.82$ (bs, 1H), 2.42 (s, 3H). HRMS calcd for $\mathrm{C}_{3} \mathrm{H}_{5} \mathrm{~N}_{2} \mathrm{OS}\left([\mathrm{M}+\mathrm{H}]^{+}\right)$117.0117, found 117.0118.

5-(噻吩-2-基)-1,3,4-噁二唑硫醇(2d): 白色固体, 收 率 90\%. m.p. $172{ }^{\circ} \mathrm{C} ;{ }^{1} \mathrm{H}$ NMR (400 MHz, DMSO- $\left.d_{6}\right) \delta$ : 14.77 (bs, 1H), 7.97 (dd, $J=5.0,1.1 \mathrm{~Hz}, 1 \mathrm{H}), 7.80$ (dd, $J=$ 3.7, $1.2 \mathrm{~Hz}, 1 \mathrm{H}$ ), 7.29 (dd, $J=4.9,3.8 \mathrm{~Hz}, 1 \mathrm{H})$. HRMS calcd for $\mathrm{C}_{6} \mathrm{H}_{5} \mathrm{~N}_{2} \mathrm{OS}_{2}\left([\mathrm{M}+\mathrm{H}]^{+}\right)$184.9838, found
184.9840.

5-(5-甲基噻吩-2-基)-1,3,4-噁二唑硫醇(2e)：黄色固 体, 收率 83\%. m.p. 200 202 ${ }^{\circ} \mathrm{C} ;{ }^{1} \mathrm{H}$ NMR (400 MHz, DMSO- $\left.d_{6}\right) \delta: 14.72(\mathrm{bs}, 1 \mathrm{H}), 7.60(\mathrm{~d}, J=3.7 \mathrm{~Hz}, 1 \mathrm{H}), 6.99$ (d, $J=3.7 \mathrm{~Hz}, 1 \mathrm{H}), 2.53$ (s, 3H). HRMS calcd for $\mathrm{C}_{7} \mathrm{H}_{7} \mathrm{~N}_{2} \mathrm{OS}_{2}\left([\mathrm{M}+\mathrm{H}]^{+}\right)$198.9994, found 198.9993 .

5-(3-甲基噻吩-2-基)-1,3,4-噁二唑硫醇(2f): 白色固 体, 收率 79\%. m.p. $177{ }^{\circ} \mathrm{C} ;{ }^{1} \mathrm{H}$ NMR (400 MHz, DMSO- $\left.d_{6}\right) \delta: 14.72(\mathrm{bs}, 1 \mathrm{H}), 7.84(\mathrm{~d}, J=5.0 \mathrm{~Hz}, 1 \mathrm{H}), 7.15$ (d, $J=5.0 \mathrm{~Hz}, 1 \mathrm{H}), 2.48$ (s, 3H). HRMS calcd for $\mathrm{C}_{7} \mathrm{H}_{7} \mathrm{~N}_{2} \mathrm{OS}_{2}\left([\mathrm{M}+\mathrm{H}]^{+}\right)$198.9994, found 198.9992 .

5-(3-溴噻吩-2-基)-1,3,4-噁二唑硫醇 (2g): 黄色固 体, 收率 83\%. m.p. 189 192 ${ }^{\circ} \mathrm{C} ;{ }^{1} \mathrm{H}$ NMR (400 MHz, DMSO- $\left.d_{6}\right) \delta: 9.92(\mathrm{~s}, 1 \mathrm{H}), 8.01(\mathrm{~d}, J=5.2 \mathrm{~Hz}, 1 \mathrm{H}), 7.37$ (d, $J=5.2 \mathrm{~Hz}, 1 \mathrm{H})$. HRMS calcd for $\mathrm{C}_{6} \mathrm{H}_{4} \mathrm{BrN}_{2} \mathrm{OS}_{2}([\mathrm{M}+$ $\mathrm{H}]^{+}$) 262.8943, found 262.8944 .

5-(吡啶-2-基)-1,3,4-噁二唑硫醇(2h): 白色固体, 收 率 81\%. m.p. 209 211 ${ }^{\circ} \mathrm{C}$ (文献值 ${ }^{[27]} 223 \sim 224{ }^{\circ} \mathrm{C}$ ); ${ }^{1} \mathrm{H}$ NMR (400 MHz, DMSO- $d_{6}$ ) $\delta: 15.03$ (bs, $1 \mathrm{H}$ ), 8.97 8.65 (m, 1H), 8.24 7.89 (m, 2H), $7.61 \sim 7.69(\mathrm{~m}, 1 \mathrm{H})$. HRMS calcd for $\mathrm{C}_{7} \mathrm{H}_{6} \mathrm{~N}_{3} \mathrm{OS}\left([\mathrm{M}+\mathrm{H}]^{+}\right) \quad 180.0226$, found 180.0224 .

5-(吡啶-3-基)-1,3,4-噁二唑硫醇(2i): 白色固体, 收 率 85\%. m.p. $230 \sim 232{ }^{\circ} \mathrm{C}$ (文献值 ${ }^{[28]} 231{ }^{\circ} \mathrm{C}$ ); ${ }^{1} \mathrm{H}$ NMR (400 MHz, DMSO- $d_{6}$ ) $\delta: 14.81$ (bs, 1H), 9.06 (d, $J=1.7$ $\mathrm{Hz}, 1 \mathrm{H}), 8.81(\mathrm{dd}, J=4.8,1.5 \mathrm{~Hz}, 1 \mathrm{H}), 8.33 \sim 8.21(\mathrm{~m}$, $1 \mathrm{H}), 7.64(\mathrm{dd}, J=8.0,4.9 \mathrm{~Hz}, 1 \mathrm{H})$. HRMS calcd for $\mathrm{C}_{7} \mathrm{H}_{6} \mathrm{~N}_{3} \mathrm{OS}\left([\mathrm{M}+\mathrm{H}]^{+}\right)$180.0226, found 180.0225 .

5-(吡啶-4-基)-1,3,4-噁二唑硫醇(2j): 黄色固体，收 率 83\%. m.p. $251 \sim 253{ }^{\circ} \mathrm{C}$ (文献值 ${ }^{[29]} 256{ }^{\circ} \mathrm{C}$ ); ${ }^{1} \mathrm{H}$ NMR $\left(400 \mathrm{MHz}\right.$, DMSO- $\left.d_{6}\right) \delta: 8.81(\mathrm{~d}, J=5.9 \mathrm{~Hz}, 2 \mathrm{H}), 7.82(\mathrm{~d}$, $J=6.0 \mathrm{~Hz}, 2 \mathrm{H})$. HRMS calcd for $\mathrm{C}_{7} \mathrm{H}_{6} \mathrm{~N}_{3} \mathrm{OS}\left([\mathrm{M}+\mathrm{H}]^{+}\right)$ 180.0226 , found 180.0224 .

\subsection{2中间体化合物 $\mathbf{6}$ 的合成}

以对羟基苯甲酸为原料，按照文献[30]步骤合成化 合物 6 的粗品.

4-(1,3,4-噁二唑-2-基)苯酚 (6a): 白色固体, 收率 90\%. m.p. $215 \sim 216{ }^{\circ} \mathrm{C} ;{ }^{1} \mathrm{H}$ NMR (400 MHz, DMSO- $d_{6}$ ) $\delta: 10.34(\mathrm{~s}, 1 \mathrm{H}), 9.23(\mathrm{~s}, 1 \mathrm{H}), 7.86(\mathrm{~d}, J=8.7 \mathrm{~Hz}, 2 \mathrm{H}), 6.96$ $(\mathrm{d}, J=8.7 \mathrm{~Hz}, 2 \mathrm{H})$. HRMS calcd for $\mathrm{C}_{8} \mathrm{H}_{7} \mathrm{~N}_{2} \mathrm{O}_{2}\left([\mathrm{M}+\mathrm{H}]^{+}\right)$ 163.0502, found 163.0503 .

4-(5-甲基-1,3,4-噁二唑-2-基)苯酚(6b)：白色固体， 收率 82\%. m.p. 232 235 ${ }^{\circ} \mathrm{C}$ (文献值 ${ }^{[31]} 236{ }^{\circ} \mathrm{C}$ ); ${ }^{1} \mathrm{H}$ NMR (400 MHz, DMSO- $\left.d_{6}\right) \delta$ : $10.28(\mathrm{~s}, 1 \mathrm{H}), 7.80$ (d, $J=$ $8.6 \mathrm{~Hz}, 2 \mathrm{H}), 6.94$ (d, $J=8.6 \mathrm{~Hz}, 2 \mathrm{H}), 2.55$ (s, 3H). HRMS 
calcd for $\mathrm{C}_{9} \mathrm{H}_{9} \mathrm{~N}_{2} \mathrm{O}_{2}\left([\mathrm{M}+\mathrm{H}]^{+}\right)$177.0659, found 177.0658.

4-(5-乙基-1,3,4-噁二唑-2-基)苯酚(6c)：粉色固体, 收率 85\%. m.p. 206 208 ${ }^{\circ} \mathrm{C}$ (文献值 ${ }^{[32]} 204 \sim 206{ }^{\circ} \mathrm{C}$ ); ${ }^{1} \mathrm{H}$ NMR (400 MHz, DMSO- $\left.d_{6}\right) \delta: 10.28(\mathrm{~s}, 1 \mathrm{H}), 7.81$ (d, $J=8.7 \mathrm{~Hz}, 2 \mathrm{H}), 6.94(\mathrm{~d}, J=8.7 \mathrm{~Hz}, 2 \mathrm{H}), 2.91$ (q, $J=7.6$ $\mathrm{Hz}, 2 \mathrm{H}), 1.31(\mathrm{t}, J=7.6 \mathrm{~Hz}, 3 \mathrm{H})$. HRMS calcd for $\mathrm{C}_{10} \mathrm{H}_{11} \mathrm{~N}_{2} \mathrm{O}_{2}\left([\mathrm{M}+\mathrm{H}]^{+}\right)$191.0815, found 191.0814.

4-(5-苯基-1,3,4-噁二唑-2-基)苯酚(6d): 白色固体, 收率 84\%. m.p. 252 253 ${ }^{\circ} \mathrm{C}$ (文献值 $\left.{ }^{[33]} 253{ }^{\circ} \mathrm{C}\right) ;{ }^{1} \mathrm{H}$ NMR (400 MHz, DMSO- $\left.d_{6}\right) \delta: 10.37(\mathrm{~s}, 1 \mathrm{H}), 8.11$ (dd, $J=$ 7.3, 2.3 Hz, 2H), 7.97 (d, $J=8.7 \mathrm{~Hz}, 2 \mathrm{H}), 7.63$ (dd, $J=8.0$, $2.5 \mathrm{~Hz}, 3 \mathrm{H}), 6.98(\mathrm{~d}, J=8.7 \mathrm{~Hz}, 2 \mathrm{H})$. HRMS calcd for $\mathrm{C}_{14} \mathrm{H}_{11} \mathrm{~N}_{2} \mathrm{O}_{2}\left([\mathrm{M}+\mathrm{H}]^{+}\right)$239.0815, found 239.0813.

\subsection{3 中间体化合物 4 的合成}

按照文献[2]的方法合成粗产物 3, 产物未经处理, 直接投入下一步. 在 $250 \mathrm{~mL}$ 圆底烧瓶中加入 3 (2.06 g, $10 \mathrm{mmol}), 100 \mathrm{~mL}$ 四氯化碳溶液, 过氧化苯甲酰(BPO) $(0.1 \mathrm{~g}, 1 \mathrm{mmol})$. 然后分批加入溴代丁二酰亚胺(NBS) (0.542 g, $9.5 \mathrm{mmol})$. 搅拌加热回流 $4 \mathrm{~h}$. 抽滤, 旋蒸除去 溶剂, 加入少量冰乙醇重结晶得中间体 4. 白色固体, 收率 89\%. m.p. 87 90 ${ }^{\circ} \mathrm{C}$ (文献值 $\left.{ }^{[34]} 87 \sim 90{ }^{\circ} \mathrm{C}\right) ;{ }^{1} \mathrm{H}$ NMR (400 MHz, $\left.\mathrm{CDCl}_{3}\right) \delta: 7.67(\mathrm{~s}, 1 \mathrm{H}), 7.53 \sim 7.47(\mathrm{~m}$, $1 \mathrm{H}), 7.37 \sim 7.33(\mathrm{~m}, 2 \mathrm{H}), 7.20 \sim 7.13(\mathrm{~m}, 1 \mathrm{H}), 4.45(\mathrm{~s}$, 2H), $3.85(\mathrm{~s}, 3 \mathrm{H}), 3.73(\mathrm{~s}, 3 \mathrm{H})$. HRMS calcd for $\mathrm{C}_{12} \mathrm{H}_{14} \mathrm{BrO}_{3}\left([\mathrm{M}+\mathrm{H}]^{+}\right)$285.0121, found 285.0122.

\subsection{4 终产物 I 和 II 的合成}

在 $100 \mathrm{~mL}$ 的圆底烧瓶中加入中间体 2 或 $6(2$ $\mathrm{mmol}), \mathrm{K}_{2} \mathrm{CO}_{3}(2.5 \mathrm{mmol}), 30 \mathrm{~mL} \mathrm{DMF}$, 搅拌 $5 \mathrm{~min}$, 向 其中加入 4 (2.2 mmol), 搅拌过夜. 将体系倒入水中, 乙 酸乙酯萃取 $(30 \mathrm{~mL} \times 3)$, 合并有机相, 饱和氯化钠溶液 洗涤, 无水硫酸钠干燥, 旋蒸除去溶剂, 柱层析 $[V($ 石油 醚) $: V$ (乙酸乙酯 $)=3: 1$ ]得终产物 I 或 II.

(E)-2-(2-\{[5-(呋喃-2-基)-1,3,4-啞二唑]-2-基硫甲基 $\}$ 苯基)-3-甲氧基丙烯酸甲酯(Ia): 淡黄色固体, 收率 74\%. m.p. 93 94 ${ }^{\circ} \mathrm{C} ;{ }^{1} \mathrm{H}$ NMR (400 MHz, DMSO-d $\left.d_{6}\right) \delta: 8.07$ (s, 1H), 7.73 (s, 1H), $7.60(\mathrm{dd}, J=5.3,3.5 \mathrm{~Hz}, 1 \mathrm{H}), 7.39 \sim$ $7.31(\mathrm{~m}, 3 \mathrm{H}), 7.16(\mathrm{dd}, J=5.3,3.5 \mathrm{~Hz}, 1 \mathrm{H}), 6.81$ (dd, $J=$ 3.1, 1.4 Hz, 1H), 4.48 (s, 2H), 3.87 (s, 3H), 3.65 (s, 3H). ${ }^{13} \mathrm{C}$ NMR (101 MHz, DMSO- $\left.d_{6}\right) \quad \delta: 166.93,163.04$, $161.16,158.05,146.94,138.27,134.91,133.19,131.56$, $129.86,127.88,114.55,112.60,108.60,61.87,51.29$, 34.75. HRMS (ESI) calcd for $\mathrm{C}_{18} \mathrm{H}_{17} \mathrm{~N}_{2} \mathrm{O}_{5} \mathrm{~S}\left([\mathrm{M}+\mathrm{H}]^{+}\right)$ 373.0853 , found 373.0856 .

(E)-2-(2-\{[5-(5-溴呋喃-2-基)-1,3,4-噁二唑]-2-基硫 甲基 $\}$ 苯基)-3-甲氧基丙烯酸甲酯(Ib): 黄色固体, 收率
79\%. m.p. 86 89 ${ }^{\circ} \mathrm{C}$; ${ }^{1} \mathrm{H}$ NMR (400 $\left.\mathrm{MHz} \mathrm{CDCl}_{3}\right) \delta$ : $7.57(\mathrm{~d}, J=2.8 \mathrm{~Hz}, 1 \mathrm{H}), 7.54 \sim 7.46(\mathrm{~m}, 1 \mathrm{H}), 7.30 \sim 7.21$ $(\mathrm{m}, 2 \mathrm{H}), 7.12(\mathrm{~m}, 1 \mathrm{H}), 7.00(\mathrm{t}, J=8.3 \mathrm{~Hz}, 1 \mathrm{H}), 6.45(\mathrm{~d}, J$ $=3.6 \mathrm{~Hz}, 1 \mathrm{H}), 4.43(\mathrm{~s}, 2 \mathrm{H}), 3.74(\mathrm{~s}, 3 \mathrm{H}), 3.64(\mathrm{~s}, 3 \mathrm{H}) ;{ }^{13} \mathrm{C}$ NMR (101 MHz, $\left.\mathrm{CDCl}_{3}\right) \delta: 167.52,164.27,160.65$, $157.40,140.73,134.47,133.00,131.58,130.02,128.27$, 128.12 , 126.47, 115.98, 114.20, 109.63, 62.02, 51.62, 35.24. HRMS (ESI) calcd for $\mathrm{C}_{18} \mathrm{H}_{16} \mathrm{BrN}_{2} \mathrm{O}_{5} \mathrm{~S}\left([\mathrm{M}+\mathrm{H}]^{+}\right)$ 450.9958 , found 450.9961 .

(E)-2-\{2-[(5-甲基-1,3,4-噁二唑)-2-基硫甲基]苯 基 \}-3-甲氧基丙烯酸甲酯(Ic): 白色固体，收率 $82 \%$. m.p. $94{ }^{\circ} \mathrm{C} ;{ }^{1} \mathrm{H}$ NMR (400 MHz, $\left.\mathrm{CDCl}_{3}\right) \delta$ : $7.58(\mathrm{~s}, 1 \mathrm{H})$, $7.54 \sim 7.46(\mathrm{~m}, 1 \mathrm{H}), 7.31 \sim 7.23(\mathrm{~m}, 2 \mathrm{H}), 7.17 \sim 7.09(\mathrm{~m}$, $1 \mathrm{H}), 4.40$ (s, 2H), 3.79 (s, 3H), 3.67 (s, 3H), 2.44 (s, 3H); ${ }^{13} \mathrm{C}$ NMR $\left(101 \mathrm{MHz}, \mathrm{CDCl}_{3}\right) \delta: 167.58,164.60,164.30$, $160.54,134.67,132.88,131.46,129.95,128.22$, 127.97, 109.68, 61.97, 51.58, 34.87, 10.88. HRMS (ESI) calcd for $\mathrm{C}_{15} \mathrm{H}_{17} \mathrm{~N}_{2} \mathrm{O}_{4} \mathrm{~S}\left([\mathrm{M}+\mathrm{H}]^{+}\right)$321.0904, found 321.0907 .

(E)-2-(2-\{[5-(噻吩-2-基)-1,3,4-啞二唑]-2-基硫甲基 $\}$ 苯基)-3-甲氧基丙烯酸甲酯(Id): 黄色固体，收率 86\%. m.p. $113 \sim 116{ }^{\circ} \mathrm{C} ;{ }^{1} \mathrm{H}$ NMR $\left(400 \mathrm{MHz}, \mathrm{CDCl}_{3}\right) \delta: 7.61$ (dd, $J=13.5,7.4 \mathrm{~Hz}, 2 \mathrm{H}), 7.54$ (dd, $J=13.9,11.1 \mathrm{~Hz}, 1 \mathrm{H})$, $7.45(\mathrm{t}, J=10.5 \mathrm{~Hz}, 1 \mathrm{H}), 7.28$ (dd, $J=18.1,14.7 \mathrm{~Hz}, 2 \mathrm{H})$, $7.22 \sim 7.12(\mathrm{~m}, 1 \mathrm{H}), 7.11 \sim 7.00(\mathrm{~m}, 1 \mathrm{H}), 4.46(\mathrm{~s}, 2 \mathrm{H})$, $3.77(\mathrm{~s}, 3 \mathrm{H}), 3.68(\mathrm{~s}, 3 \mathrm{H}) ;{ }^{13} \mathrm{C} \mathrm{NMR}\left(101 \mathrm{MHz}, \mathrm{CDCl}_{3}\right) \delta$ : $167.68,163.93,161.88,160.68,134.70,132.96,131.59$, $130.16,130.13,129.61,128.36,128.20,128.17,124.76$, 109.73, 62.10, 51.74, 35.20. HRMS calcd for $\mathrm{C}_{18} \mathrm{H}_{17} \mathrm{~N}_{2}$ $\mathrm{O}_{4} \mathrm{~S}_{2}\left([\mathrm{M}+\mathrm{H}]^{+}\right)$389.0624, found 389.0626.

(E)-2-(2-\{[5-(5-甲基噻吩-2-基)-1,3,4-啞二唑]-2-基 硫甲基 苯基)-3-甲氧基丙烯酸甲酯(Ie): 黄色固体, 收 率 72\%. m.p. $125 \sim 127{ }^{\circ} \mathrm{C} ;{ }^{1} \mathrm{H}$ NMR (400 MHz, $\mathrm{CDCl}_{3}$ ) $\delta: 7.59 \sim 7.46(\mathrm{~m}, 1 \mathrm{H}), 7.49 \sim 7.39(\mathrm{~m}, 1 \mathrm{H}), 7.39 \sim 7.27$ $(\mathrm{m}, 1 \mathrm{H}), 7.25 \sim 7.12(\mathrm{~m}, 2 \mathrm{H}), 7.13 \sim 6.93(\mathrm{~m}, 1 \mathrm{H}), 6.76 \sim$ $6.52(\mathrm{~m}, 1 \mathrm{H}), 4.33(\mathrm{~s}, 2 \mathrm{H}), 3.76(\mathrm{~m}, 3 \mathrm{H}), 3.67(\mathrm{~m}, 3 \mathrm{H})$, $2.40(\mathrm{~s}, 3 \mathrm{H}) ;{ }^{13} \mathrm{C}$ NMR (101 $\left.\mathrm{MHz}, \mathrm{CDCl}_{3}\right) \delta:$ 167.70, $163.51,161.86,160.64,145.51,134.73,132.91,131.54$, $130.12,129.84,128.35,128.12,126.60,122.28,109.76$, $62.09,51.73,35.18$, 15.50. HRMS (ESI) calcd for $\mathrm{C}_{19} \mathrm{H}_{19} \mathrm{~N}_{2} \mathrm{O}_{4} \mathrm{~S}_{2}\left([\mathrm{M}+\mathrm{H}]^{+}\right)$403.0781, found 403.0782.

(E)-2-(2-\{[5-(3-甲基噻吩-2-基)-1,3,4-啞二唑]-2-基 硫甲基 苯基)-3-甲氧基丙烯酸甲酯(If): 黄色固体, 收 率 78\%. m.p. $107 \sim 110{ }^{\circ} \mathrm{C} ;{ }^{1} \mathrm{H} \mathrm{NMR}\left(400 \mathrm{MHz}, \mathrm{CDCl}_{3}\right)$ $\delta: 7.60(\mathrm{~s}, 1 \mathrm{H}), 7.57$ (dd, $J=10.5,5.6 \mathrm{~Hz}, 1 \mathrm{H}), 7.31$ (d, $J=$ $4.8 \mathrm{~Hz}, 1 \mathrm{H}), 7.31 \sim 7.23(\mathrm{~m}, 2 \mathrm{H}), 7.14(\mathrm{~m}, 1 \mathrm{H}), 6.89(\mathrm{~d}$, 
$J=4.9 \mathrm{~Hz}, 1 \mathrm{H}), 4.45$ (s, 2H), 3.76 (s, 3H), 3.67 (s, 3H), 2.53 (s, 3H); ${ }^{13} \mathrm{C}$ NMR (101 MHz, $\left.\mathrm{CDCl}_{3}\right) \delta: 167.66$, $163.25,162.37,160.64,141.84,134.80,132.93,131.71$, $131.57,130.12,128.57,128.34,128.11,118.97,109.76$, $62.08,51.72,35.18$, 15.90. HRMS (ESI) calcd for $\mathrm{C}_{19} \mathrm{H}_{19} \mathrm{~N}_{2} \mathrm{O}_{4} \mathrm{~S}_{2}\left([\mathrm{M}+\mathrm{H}]^{+}\right)$403.0781, found 403.0784.

(E)-2-(2-\{[5-(3-溴噻吩-2-基)-1,3,4-噁二唑]-2-基硫 甲基\} 苯基)-3-甲氧基丙烯酸甲酯(Ig): 黄色固体, 收率 76\%. m.p. 93 95 ${ }^{\circ} \mathrm{C} ;{ }^{1} \mathrm{H}$ NMR $\left(400 \mathrm{MHz}, \mathrm{CDCl}_{3}\right) \delta$ : $7.62(\mathrm{~s}, 1 \mathrm{H}), 7.61 \sim 7.53(\mathrm{~m}, 1 \mathrm{H}), 7.42(\mathrm{~d}, J=5.1 \mathrm{~Hz}, 1 \mathrm{H})$, $7.35 \sim 7.25(\mathrm{~m}, 2 \mathrm{H}), 7.22 \sim 7.12(\mathrm{~m}, 1 \mathrm{H}), 7.08(\mathrm{~d}, J=5.1$ $\mathrm{Hz}, 1 \mathrm{H}), 4.48$ (s, 2H), 3.76 (s, 3H), 3.68 (s, 3H); ${ }^{13} \mathrm{C}$ NMR $\left(101 \mathrm{MHz}, \mathrm{CDCl}_{3}\right) \delta: 167.67,164.47,160.66,160.52$, $134.62,132.94,132.68,131.60,130.13,129.98,128.39$, 128.19, 120.81, 113.43, 109.77, 62.14, 51.78, 35.22. HRMS (ESI) calcd for $\mathrm{C}_{18} \mathrm{H}_{16} \mathrm{BrN}_{2} \mathrm{O}_{4} \mathrm{~S}_{2}\left([\mathrm{M}+\mathrm{H}]^{+}\right)$ 466.9729, found 466.9728 .

(E)-2-(2-\{[5-(吡啶-2-基)-1,3,4-噁二唑]-2-基硫甲基 $\}$ 苯基)-3-甲氧基丙烯酸甲酯(Ih): 黄色油状, 收率 71\%. ${ }^{1} \mathrm{H}$ NMR (400 MHz, $\left.\mathrm{CDCl}_{3}\right) \delta: 8.71(\mathrm{~d}, J=4.4 \mathrm{~Hz}, 1 \mathrm{H})$, $8.14(\mathrm{~d}, J=7.9 \mathrm{~Hz}, 1 \mathrm{H}), 7.78$ (ddd, $J=26.8,16.6,10.3 \mathrm{~Hz}$, $1 \mathrm{H}), 7.72 \sim 7.54(\mathrm{~m}, 2 \mathrm{H}), 7.38(\mathrm{dd}, J=7.0,5.1 \mathrm{~Hz}, 1 \mathrm{H})$, $7.36 \sim 7.23(\mathrm{~m}, 2 \mathrm{H}), 7.25 \sim 7.06(\mathrm{~m}, 1 \mathrm{H}), 4.49(\mathrm{~s}, 2 \mathrm{H})$, $3.75(\mathrm{~s}, 3 \mathrm{H}), 3.68(\mathrm{~s}, 3 \mathrm{H}) ;{ }^{13} \mathrm{C} \mathrm{NMR}\left(101 \mathrm{MHz}, \mathrm{CDCl}_{3}\right) \delta$ : 167.53, 166.04, 164.71, 160.60, 150.14, 143.08, 137.25, 134.46, 133.01, 131.51, 130.14, 128.26, 128.11, 125.75, $122.68,109.64,62.00,51.63,34.99$. HRMS (ESI) calcd for $\mathrm{C}_{19} \mathrm{H}_{18} \mathrm{~N}_{3} \mathrm{O}_{4} \mathrm{~S}\left([\mathrm{M}+\mathrm{H}]^{+}\right)$384.1013, found 384.1015.

(E)-2-(2-\{[5-(吡啶-3-基)-1,3,4-噁二唑]-2-基硫甲基 $\}$ 苯基)-3-甲氧基丙烯酸甲酯(Ii): 黄色油状, 收率 73\%. m.p. $107 \sim 110{ }^{\circ} \mathrm{C} ;{ }^{1} \mathrm{H}$ NMR (400 MHz, $\left.\mathrm{CDCl}_{3}\right) \delta$ : 9.17 (s, $1 \mathrm{H}), 8.70(\mathrm{~d}, J=3.8 \mathrm{~Hz}, 1 \mathrm{H}), 8.31-8.09(\mathrm{~m}, 1 \mathrm{H}), 7.64(\mathrm{~s}$, 1H), 7.57 (dd, $J=6.5,2.4 \mathrm{~Hz}, 1 \mathrm{H}), 7.37$ (dt, $J=14.9,7.5$ $\mathrm{Hz}, 1 \mathrm{H}), 7.29$ (m, 2H), 7.18 (dd, $J=6.6,2.3 \mathrm{~Hz}, 1 \mathrm{H}), 4.50$ (s, 2H), 3.80 (s, 3H), 3.68 (s, 3H); ${ }^{13} \mathrm{C}$ NMR (101 MHz, $\left.\mathrm{CDCl}_{3}\right) \delta: 167.47,165.26,163.46,160.61,152.20,147.39$, $134.55,133.65,132.93,131.54,129.94,128.21,128.05$, $123.77,120.03,109.58,61.99,51.59,35.07$. HRMS (ESI) calcd for $\mathrm{C}_{19} \mathrm{H}_{18} \mathrm{~N}_{3} \mathrm{O}_{4} \mathrm{~S}\left([\mathrm{M}+\mathrm{H}]^{+}\right)$384.1013, found 384.1014.

(E)-2-(2-\{[5-(吡啶-4-基)-1,3,4-噁二唑]-2-基硫甲基 $\}$ 苯基)-3-甲氧基丙烯酸甲酯( $\mathbf{I j})$ : 黄色固体, 收率 $83 \%$. m.p. 102 104 ${ }^{\circ} \mathrm{C} ;{ }^{1} \mathrm{H}$ NMR (400 MHz, $\mathrm{CDCl}_{3}$ ) $\delta: 8.62$ (d, $J=5.5 \mathrm{~Hz}, 2 \mathrm{H}), 7.67$ (d, $J=5.8 \mathrm{~Hz}, 2 \mathrm{H}), 7.49$ (s, 1H), $7.47 \sim 7.36(\mathrm{~m}, 1 \mathrm{H}), 7.25 \sim 7.10(\mathrm{~m}, 2 \mathrm{H}), 7.11 \sim 6.95(\mathrm{~m}$,
1H), 4.37 (s, 2H), 3.69 (s, 3H), 3.56 (s, 3H); ${ }^{13} \mathrm{C}$ NMR $\left(101 \mathrm{MHz}, \mathrm{CDCl}_{3}\right) \delta: 167.50,166.05,163.69,160.61$, $150.70,134.36,132.89,131.52,130.53,129.94,128.22$, $128.09,119.88,109.54,62.00,51.59,35.01$. HRMS (ESI) calcd for $\mathrm{C}_{19} \mathrm{H}_{18} \mathrm{~N}_{3} \mathrm{O}_{4} \mathrm{~S}\left([\mathrm{M}+\mathrm{H}]^{+}\right)$384.1013, found 384.1016.

(E)-2-\{2-[4-(噁二唑-2-基)苯基硫甲基]苯基\}-3-甲氧 基丙烯酸甲酯(IIa): 黄色固体, 收率 87\%. m.p. 125 $127{ }^{\circ} \mathrm{C} ;{ }^{1} \mathrm{H}$ NMR $\left(400 \mathrm{MHz}, \mathrm{CDCl}_{3}\right) \delta: 8.41(\mathrm{~s}, 1 \mathrm{H}), 7.98$ $-7.90(\mathrm{~m}, 2 \mathrm{H}), 7.62(\mathrm{~s}, 1 \mathrm{H}), 7.51(\mathrm{~s}, 1 \mathrm{H}), 7.29(\mathrm{~d}, J=24.9$ $\mathrm{Hz}, 2 \mathrm{H}), 7.17(\mathrm{~d}, J=27.1 \mathrm{~Hz}, 1 \mathrm{H}), 7.00(\mathrm{~d}, J=7.9 \mathrm{~Hz}$, 2H), 5.05 (s, 2H), 3.77 (d, $J=19.4 \mathrm{~Hz}, 3 \mathrm{H}), 3.70$ (s, 3H); ${ }^{13} \mathrm{C}$ NMR $\left(101 \mathrm{MHz}, \mathrm{CDCl}_{3}\right) \delta: 167.70,164.51,161.63$, $160.35,152.47,135.28,131.39,131.21,128.74,128.11$, $127.79,127.36,115.95,115.38,109.73,68.17,62.08$, 51.69. HRMS (ESI) calcd for $\mathrm{C}_{20} \mathrm{H}_{19} \mathrm{~N}_{2} \mathrm{O}_{5}\left([\mathrm{M}+\mathrm{H}]^{+}\right)$ 367.1288 , found 367.1285 .

(E)-2-\{2-[4-(5-甲基噁二唑-2-基)苯基硫甲基]苯 基\}-3-甲氧基丙烯酸甲酯(IIb): 黄色固体，收率 $85 \%$. m.p. $116 \sim 119{ }^{\circ} \mathrm{C} ;{ }^{1} \mathrm{H}$ NMR (400 MHz, $\left.\mathrm{CDCl}_{3}\right) \delta$ : $7.93-$ $7.74(\mathrm{~m}, 2 \mathrm{H}), 7.54(\mathrm{~s}, 1 \mathrm{H}), 7.51 \sim 7.39(\mathrm{~m}, 1 \mathrm{H}), 7.34-$ $7.20(\mathrm{~m}, 2 \mathrm{H}), 7.21 \sim 7.09(\mathrm{~m}, 1 \mathrm{H}), 6.94(\mathrm{t}, J=13.8 \mathrm{~Hz}$, 2H), 4.99 (s, 2H), 3.70 (s, 3H), 3.66 (s, 3H), 2.45 (s, 3H); ${ }^{13} \mathrm{C}$ NMR $\left(101 \mathrm{MHz}, \mathrm{CDCl}_{3}\right) \delta: 167.63,164.65,163.12$, $161.32,160.31,135.37,131.39,131.19,128.31,128.04$, 127.69, 127.29, 116.43, 115.29, 109.73, 68.12, 62.01, $51.60,10.89$. HRMS(ESI) calcd for $\mathrm{C}_{21} \mathrm{H}_{21} \mathrm{~N}_{2} \mathrm{O}_{5}([\mathrm{M}+\mathrm{H}]$ $\left.{ }^{+}\right) 381.1445$, found 381.1444 .

(E)-2-\{2-[4-(5-乙基啞二唑-2-基)苯基硫甲基]苯 基 \}-3-甲氧基丙烯酸甲酯(IIc): 黄色固体，收率 $83 \%$. m.p. $124 \sim 125{ }^{\circ} \mathrm{C} ;{ }^{1} \mathrm{H}$ NMR (400 MHz, $\mathrm{CDCl}_{3}$ ) $\delta: 7.79$ (d, $J=11.1 \mathrm{~Hz}, 2 \mathrm{H}), 7.47(\mathrm{~s}, 1 \mathrm{H}), 7.46 \sim 7.33(\mathrm{~m}, 1 \mathrm{H})$, $7.28 \sim 7.15(\mathrm{~m}, 2 \mathrm{H}), 7.13 \sim 7.02(\mathrm{~m}, 1 \mathrm{H}), 6.91(\mathrm{dd}, J=$ 28.5, $8.7 \mathrm{~Hz}, 2 \mathrm{H}), 4.93$ (s, 2H), 3.68 (s, 3H), 3.59 (s, 3H), $2.78(\mathrm{q}, J=7.6 \mathrm{~Hz}, 2 \mathrm{H}), 1.37 \sim 1.22(\mathrm{~m}, 3 \mathrm{H}) ;{ }^{13} \mathrm{C} \mathrm{NMR}$ $\left(101 \mathrm{MHz}, \mathrm{CDCl}_{3}\right) \delta: 167.72,167.26,164.59,161.32$, $160.28,135.36,131.31,131.18,128.40,128.13,127.76$, 127.35, 116.61, 115.29, 109.82, 68.15, 62.08, 51.71, 19.13, 10.89. HRMS (ESI) calcd for $\mathrm{C}_{22} \mathrm{H}_{23} \mathrm{~N}_{2} \mathrm{O}_{5}\left([\mathrm{M}+\mathrm{H}]^{+}\right)$ 395.1601 , found 395.1605 .

(E)-2-\{2-[4-(5-苯基噁二唑-2-基)苯基硫甲基]苯 基\}-3-甲氧基丙烯酸甲酯(IId): 黄色固体, 收率 88\%. m.p. $154 \sim 157{ }^{\circ} \mathrm{C} ;{ }^{1} \mathrm{H}$ NMR $\left(400 \mathrm{MHz}, \mathrm{CDCl}_{3}\right) \delta: 8.17 \sim$ $8.06(\mathrm{~m}, 2 \mathrm{H}), 8.01(\mathrm{~d}, J=9.2 \mathrm{~Hz}, 2 \mathrm{H}), 7.60(\mathrm{~s}, 1 \mathrm{H}), 7.47 \sim$ $7.54(\mathrm{~m}, 4 \mathrm{H}), 7.31 \sim 7.36(\mathrm{~m}, 2 \mathrm{H}), 7.17 \sim 7.23(\mathrm{~m}, 1 \mathrm{H})$, 
$7.02(\mathrm{~d}, J=8.8 \mathrm{~Hz}, 2 \mathrm{H}), 5.05$ (s, 2H), 3.82 (s, 3H), 3.69 (s, $3 \mathrm{H}) ;{ }^{13} \mathrm{C} \mathrm{NMR}\left(101 \mathrm{MHz}, \mathrm{CDCl}_{3}\right) \delta: 167.83,164.55$, $164.13,161.59,160.29,135.33,131.59,131.29,131.21$, $129.07,128.67,128.22,127.84,127.42,126.81,124.03$, 116.40, 115.42, 109.92, 68.24, 62.14, 51.80. HRMS (ESI) calcd for $\mathrm{C}_{26} \mathrm{H}_{23} \mathrm{~N}_{2} \mathrm{O}_{5}\left([\mathrm{M}+\mathrm{H}]^{+}\right)$443.1601, found 443.1605 .

\subsection{5 终产物 III 的合成}

在 $50 \mathrm{~mL}$ 单口烧瓶中加入 I (1 mmol), 二氯甲烷 20 $\mathrm{mL}$, 搅拌溶解, $0{ }^{\circ} \mathrm{C}$ 下向体系内滴加 MCPBA (1.6 $\mathrm{mmol}$ ), 保持 $0{ }^{\circ} \mathrm{C} 反$ 应 $2 \mathrm{~h}$, 升至室温反应 $5 \mathrm{~h}$, 薄层色谱 (TLC) 监测反应进行. 反应结束, 用 $0.25 \mathrm{~mol} / \mathrm{L}$ 的 $\mathrm{Na}_{2} \mathrm{HPO}_{4}$ 溶液洗涤, $\mathrm{Na}_{2} \mathrm{SO}_{4}$ 干燥, 旋蒸除去溶剂, 柱层 析分离.

(E)-2-(2-\{[5-(呋喃-2-基)-1,3,4-啞二唑]-2-基亚砜基 甲基 苯基)-3-甲氧基丙烯酸甲酯(IIIa): 白色固体, 收 率 67\%. m.p. 134 $135{ }^{\circ} \mathrm{C} ;{ }^{1} \mathrm{H}$ NMR (400 MHz, $\mathrm{CDCl}_{3}$ ) $\delta: 7.69(\mathrm{~s}, 1 \mathrm{H}), 7.63(\mathrm{~s}, 1 \mathrm{H}), 7.47 \sim 7.24(\mathrm{~m}, 4 \mathrm{H}), 7.20(\mathrm{~d}$, $J=7.4 \mathrm{~Hz}, 1 \mathrm{H}), 6.73 \sim 6.56(\mathrm{~m}, 1 \mathrm{H}), 4.55 \sim 4.77(\mathrm{~m}, 2 \mathrm{H})$, $3.82(\mathrm{~s}, 3 \mathrm{H}), 3.68(\mathrm{~s}, 3 \mathrm{H}) ;{ }^{13} \mathrm{C} \mathrm{NMR}\left(101 \mathrm{MHz}, \mathrm{CDCl}_{3}\right) \delta$ : $167.39,165.30,161.01,159.54,147.00,138.28,133.68$, $132.03,131.15,129.12,128.46,127.67,116.37,112.68$, 109.37, 62.25, 59.30, 51.82. HRMS (ESI) calcd for $\mathrm{C}_{18} \mathrm{H}_{17} \mathrm{~N}_{2} \mathrm{O}_{6} \mathrm{~S}\left([\mathrm{M}+\mathrm{H}]^{+}\right)$389.0802, found 389.0804.

(E)-2-(2-\{[5-(5-溴呋喃-2-基)-1,3,4-噁二唑]-2-基亚 砜基甲基 苯基)-3-甲氧基丙烯酸甲酯(IIIb): 黄色油状, 收率 60\%. ${ }^{1} \mathrm{H}$ NMR (400 MHz, $\left.\mathrm{CDCl}_{3}\right) \delta: 7.55$ (s, 1H), $7.33(\mathrm{~d}, J=7.0 \mathrm{~Hz}, 1 \mathrm{H}), 7.29 \sim 7.24(\mathrm{~m}, 1 \mathrm{H}), 7.23 \sim 7.17$ $(\mathrm{m}, 1 \mathrm{H}), 7.16 \sim 7.05(\mathrm{~m}, 2 \mathrm{H}), 6.50(\mathrm{~d}, J=3.4 \mathrm{~Hz}, 1 \mathrm{H})$, $4.50 \sim 4.71(\mathrm{~m}, 2 \mathrm{H}), 3.72(\mathrm{~s}, 3 \mathrm{H}), 3.59(\mathrm{~s}, 3 \mathrm{H}) ;{ }^{13} \mathrm{C} \mathrm{NMR}$ $\left(101 \mathrm{MHz}, \mathrm{CDCl}_{3}\right) \delta: 167.25,165.39,161.03,158.41$, $139.88,133.68,131.99,131.07,129.02,128.32,128.06$, 127.66, 118.41, 114.73, 109.16, 62.19, 59.20, 51.71. HRMS (ESI) calcd for $\mathrm{C}_{18} \mathrm{H}_{16} \mathrm{BrN}_{2} \mathrm{O}_{6} \mathrm{~S}\left([\mathrm{M}+\mathrm{H}]^{+}\right)$ 466.9907, found 466.9909.

\subsection{6 目标化合物的抑菌活性测试}

杀菌活性测试采用菌丝生长速率抑制法 ${ }^{[35,36]}$, 选择 PDA 作为培养基, 将目标化合物用滴入两滴 DMSO 的 蒸馏水配制成 $500 \mu \mathrm{g} / \mathrm{ml}$ 溶液. 取 $1 \mathrm{~mL}$ 与 $9 \mathrm{~mL}$ PDA 混 合均匀, 待其凝固之后, 于培养基中心接种直径 $4 \mathrm{~mm}$ 的真菌菌种, 置于 $28{ }^{\circ} \mathrm{C}$ 恒温培养箱中培养 $48 \mathrm{~h}$. 对于 生长缓慢的菌种可适当延长培养时间. $48 \mathrm{~h}$ 后, 十字交 叉法测量菌落直径, 与空白对照进行比对. 菌丝生长抑 制率 $(\%)=($ 空白对照菌落增长直径一药剂处理菌落增 长直径)/空白对照菌落增长直径 $\times 100 \%$.
3.2.7 目标化合物的毒力回归方程及 $\mathrm{EC}_{50}$ 计算 采用 DPSv 7.05 软件计算毒力回归方程值 ${ }^{[37]}$.

辅助材料(Supporting Information) 化合物 $\mathbf{I a} \sim \mathbf{I j}$, IIa $\sim$ IId, IIIa $\sim$ IIIb 的 ${ }^{1} \mathrm{H}$ NMR 和 ${ }^{13} \mathrm{C} \mathrm{NMR}$ 原始谱图. 这些材料可以免费从本刊网站(http://sioc-journal.cn/)上 下载.

\section{References}

[1] Chai, B.-S.; Wang, S.-Y.; Yu, W.-Q.; Li, H.-C.; Song, C.-J.; Xu, Y.; Liu, C.-L.; Chang, J.-B. Bioorg. Med. Chem. Lett. 2013, 23, 3505.

[2] Huang, W.; Zhao, P.-L.; Liu, C.-L.; Chen, Q.; Liu, Z.-M.; Yang, G.-F. J. Agric. Food Chem. 2007, 55, 3004.

[3] Sauter, H.; Steglich, W.; Anke, T. Angew. Chem., Int. Ed. 1999, 38, 1328.

[4] Chen, H.-S.; Li, Z.-M.; Han, Y.-F. J. Agric. Food Chem. 2000, 48, 5312.

[5] Singh, N.; Sangwan, N. K.; Dhindsa, K. S. Pest Manage. Sci. 2000 $56,284$.

[6] Tanitame, A.; Oyamada, Y.; Ofuji, K.; Fujimoto, M.; Iwai, N.; Hiyama, Y.; Suzuki, K.; Ito, H.; Terauchi, H.; Kawasaki, M. J. Med Chem. 2004, 47, 3693.

[7] Bekhit, A. A.; Ashour, H.; Guemei, A. A. Arch. Pharm. 2005, 338, 167.

[8] Fan, Z.-J.; Yang, Z.-K.; Zhang, H.-K.; Mi, N.; Wang, H.; Cai, F.; Zuo, X.; Zheng, Q.-X.; Song, H.-B. J. Agric. Food Chem. 2009, 58, 2630.

[9] Dickinson, R. P.; Bell, A. S.; Hitchcock, C. A.; Narayanaswami, S.; Ray, S. J.; Richardson, K.; Troke, P. F. Bioorg. Med. Chem. Lett. 1996, 6, 2031.

[10] Onodera, J.; Sato, S.; Kumazawa, S.; Ito, A.; Saishoji, S.; Niizeki, Y. JP 96127568, 1996 [Chem. Abstr. 1996, 125, 142713].

[11] Li, Y.; Zhang, H.-Q.; Liu, J.; Yang, X.-P.; Liu, Z.-J. J. Agric. Food Chem. 2006, 54, 3636.

[12] Park, H.-J.; Lee, K.; Park, S.-J.; Ahn, B.; Lee, J.-C.; Cho, H.; Lee, K.-I. Bioorg. Med. Chem. Lett. 2005, 15, 3307.

[13] Naito, H.; Ohsuki, S.; Atsumi, R.; Minami, M.; Mochizuki, M.; Hirotani, K.; Kumazawa, E.; Ejima, A. Chem. Pharm. Bull. 2005, 53, 153.

[14] Tu, S.; Xie, Y.-Q.; Gui, S.-Z.; Ye, L.-Y.; Huang, Z.-L.; Huang, Y.-B.; Che, L.-M. Bioorg. Med. Chem. Lett. 2014, 24, 2173.

[15] Liu, J.-C; Wang, W.-D; He, H.-W. Chin. J. Org. Chem. 2014, 37, 1447 (in Chinese).

(刘建超, 王卫东, 贺红武, 有机化学, 2014, 37, 1447.)

[16] Xu, G.-F.; Song, B.-A.; Bhadury, P. S.; Yang, S.; Zhang, P.-Q.; Jin, L.-H.; Xue, W.; Hu, D.-Y.; Lu, P. Bioorg. Med. Chem. 2007, 15, 3768.

[17] Rajput, A.; Kankhare, A. Pharma Chem. 2015, 7, 143

[18] Wu, X.-L.; Zhu, C.-F.; Lv, Z.-D.; Wei, C.-S.; Liao, C.-C. Chin. J. Org. Chem. 2011, 31, 824 (in Chinese).

(武现丽, 朱春风, 吕志丹, 魏成事, 廖新成, 有机化学, 2011, 31, 824.)

[19] Luo, X.-Q. M.S. Thesis, Guizhou University, Guiyang, 2008 (in Chinese). (罗小琼, 硕士论文, 贵州大学, 贵阳, 2008.)

[20] Chen, M. M.S. Thesis, Nanjing Agriculture University, Nanjing, 2011 (in Chinese).

(陈敏, 硕士论文, 南京农业大学, 南京, 2011.)

[21] Marty, M. S. R.; Rnaelingam, T. Indian J. Chem., Sect. B 1988, 
27B. 293.

[22] Paehhmaia, V. L.; Parikh, A. R. J. Indian Chem. Soc. 1988, 65. 357.

[23] Li, Q.-Z; Song, B.-A; Chen, J. Pesticide 2005, 44. 538 (in Chinese). (李黔柱, 宋宝安, 陈江, 刘杰, 杨松, 胡德禹, 金林红, 农药, 2005, 44. 538.)

[24] Jha, K. K.; Samad, A.; Kumar, Y.; Shaharyar, M.; Khosa, R. L.; Jain, J.; Kumar, V.; Singh, P. Eur. J. Med. Chem. 2010, 45, 4963.

[25] Basoglu, S.; Yolal, M.; Demirci, S.; Demirbas, N.; Bektas, H.; Karaoglu, S. Acta Pol. Pharm. 2013, 70, 229.

[26] Liu, X.-H.; Chen, P.-Q.; Wang, B.-L.; Dong, W.-L. Chem. Biol. Drug Des. 2010, 75, 228.

[27] Ahmad, R.; Iqbal, R.; Akhtar, H.; Haq, Z.; Duddeck, H.; Stefaniak, L.; Sitkowski, J. Nucleosides, Nucleotides Nucleic Acids 2001, 20, 1671.

[28] Patel, M.; Modi, N.; Raval, J.; Menon, S. Org. Biomol. Chem. 2012, 10,1785 .

[29] Rao, M.; Rajurkar, V. Asian J. Chem. 2011, 23, 2648.

[30] Sławiński, J.; Pogorzelska, A.; Żołnowska, B.; Brożewicz, K.; Vul- lo, D.; Supuran, C. T. Eur. J. Med. Chem. 2014, 82, 47.

[31] Sadek, B.; Fahelelbom, K. Molecules 2011, 16, 4339.

[32] Arnold, L. D.; Coe, J. W.; Kaneko, T.; Moyer, M. P. US 6130217, 2000 [Chem. Abstr. 2000, 133, 296449].

[33] Yildirim, N.; Bekircan, O. J. Chem. Res. 2013, 37, 160.

[34] Cliff, G. R.; Richards; I. C. EP 378308, 1990 [Chem. Abstr. 1991, 114, 81863].

[35] Chen, Y.-W.; Wan, Y.-Y.; Liu, Q.-X.; Liu, J.-B.; Xiong, L.-X.; Yu, S.-J.; Li, Z.-M. Chin. J. Org. Chem. 2015, 35, 882 (in Chinese). (陈有为, 万䒯䒯, 刘巧霞, 刘敬波, 熊丽霞, 于淑晶, 李正名, 有机化学, 2015, 35, 882.)

[36] Chen, N.-C. Bioassay Technology for Pestcides, Beijing Agricultural University Press, Beijing, 1991, p. 161 (in Chinese).

(陈春年，农药生物技术测定，北京农业大学出版社，北京， 1991, p. 161.)

[37] Li, Y.; Gao, Z.-S.; Li, J.-T.; Liu, F. J. Peanut Sci. 2012, 41, 13 (in Chinese).

(李杨, 高志山, 李建涛, 刘峰, 花生学报, 2012, 41, 13.)

(Zhao, X.) 